\title{
Homogenization of Magnetodielectric Photonic Crystals
}

\author{
A. A. Krokhin ${ }^{1,2}$ and E. Reyes ${ }^{2}$ \\ ${ }^{1}$ Department of Physics, University of North Texas, P.O. Box 311427, Denton, Texas 76203, USA \\ ${ }^{2}$ Instituto de Física, Universidad Autónoma de Puebla, Apartado Postal J-48, Puebla 72570, Mexico
}

(Received 30 July 2003; published 9 July 2004)

\begin{abstract}
We calculate the low-frequency index of refraction of a medium which is homogeneous along axis $z$ and possesses a periodic dependence of the permittivity $\varepsilon(\mathbf{r})$ and permeability $\mu(\mathbf{r})$ in the $x-y$ plane $(2 \mathrm{D}$ magnetodielectric photonic crystal). Exact analytical formulas for the effective index of refraction for two eigenmodes with vector $\mathbf{E}$ or $\mathbf{H}$ polarized along axis $z$ are obtained. We show that, unlike nonmagnetic photonic crystals where the $E$ mode is ordinary and the $H$ mode is extraordinary, now both modes exhibit extraordinary behavior. Because of this distinction, the magnetodielectric photonic crystals exhibit optical properties that do not exist for natural crystals. We also discuss the limiting case of perfectly conducting cylinders and clarify the so-called problem of noncommuting limits, $\omega \rightarrow 0$ and $\varepsilon \rightarrow \infty$.
\end{abstract}

DOI: 10.1103/PhysRevLett.93.023904

PACS numbers: 42.70.Qs, 41.20.Jb, 42.25.Lc

Magnetodielectric photonic crystals (MDPCs) - periodically arranged composites made up of magnetic semiconductors (or dielectrics) - represent a new class of artificial materials [1] with tunable by external magnetic field [2] band gap. In the low-frequency limit $(\omega \rightarrow 0)$, the wavelength covers many lattice periods thus repeating the situation that exists in the optics of natural crystals when the wavelength of visible light is much larger than atomic spacing. Atoms of any transparent natural crystal do not exhibit noticeable magnetic susceptibility in the presence of light; therefore they are considered to be nonmagnetic. As a result, all equations of crystal optics have been derived for nonmagnetic $\left(\mu_{i k}=\delta_{i k}\right)$ materials [3]. Recently Smith and Schurig [4] have shown that the electrodynamics of the left-hand medium requires introduction of the tensor $\mu_{i k}$ in order to describe adequately the effects of macroscopic magnetic inclusions. Inclusions in a MDPC (with either positive or negative permeability) are macroscopic magnetically susceptible "atoms." Metallic inclusions in a dielectric medium may give rise to the effective magnetic permeability at optical frequencies [5]. Recently, magnetodielectric photonic nanocrystals have been fabricated [6]. A review of the properties of MDPCs is given in Ref. [7].

In this Letter we calculate the effective permittivity and permeability of MDPCs and demonstrate that optics of such a periodic medium turn out to be different from the optics of natural crystals. In a natural crystal, since its magnetic susceptibility is negligible, optical anisotropy is due only to the dielectric tensor $\varepsilon_{i k}$. As a result, the eigenmodes of Maxwell equations in crystals are classified as "ordinary" and "extraordinary" waves. The ordinary wave exhibits higher symmetry as compared to the extraordinary wave. In particular, its index of refraction is independent on the direction of propagation. In what follows we show that, in an artificial medium with macroscopic magnetic atoms, the tensor $\mu_{i k} \neq \delta_{i k}$ and optical anisotropy are determined by both tensors $\varepsilon_{i k}$ and $\mu_{i k}$. Therefore both eigenmodes turn out to be extraordinary waves; i.e., their indices of refraction depend on the direction of propagation.

To calculate the effective index of refraction

$$
n_{\mathrm{eff}}(\hat{\mathbf{k}})=\lim _{k \rightarrow 0} \frac{c k}{\omega},
$$

where $\hat{\mathbf{k}}=\mathbf{k} / k$ is a unit vector in the direction of propagation, one needs to consider the low-frequency limit in the wave equation for inhomogeneous medium. Numerical calculations [1,2] show that the lowest (acoustic) branch of the dispersion relation exhibits almost linear behavior from $k=0$ to the middle of the Brillouin zone. Therefore the index of refraction (1) is valid not only in the limit $k a \ll 1$ ( $a$ is the lattice period), but for much shorter wavelength $k a \leq 0.5$ as well.

For a 2D photonic crystal, the equations for the $E$ - and $H$-polarized modes have the following form:

$$
\begin{aligned}
& \nabla \cdot\left[\mu^{-1}(\mathbf{r}) \nabla E\right]=-(\omega / c)^{2} \varepsilon(\mathbf{r}) E, \\
& \nabla \cdot\left[\varepsilon^{-1}(\mathbf{r}) \nabla H\right]=-(\omega / c)^{2} \mu(\mathbf{r}) H .
\end{aligned}
$$

These equations are symmetric with respect to the replacement $E \leftrightarrow H$ and $\varepsilon \leftrightarrow \mu$. Therefore, in what follows, we calculate $n_{\text {eff }}$ for the $H$ mode only and the effective index of refraction for the $E$ mode is obtained from that for the $H$ mode by interchanging $\varepsilon$ and $\mu$, i.e., $n_{\text {eff }}^{(E)}\{\varepsilon(\mathbf{r}), \mu(\mathbf{r})\} \equiv n_{\text {eff }}^{(H)}\{\mu(\mathbf{r}), \varepsilon(\mathbf{r})\}$. Even without further calculations, it is clear from Eqs. (2) and (3) that the $E$ and $H$ modes possess the same symmetry in a MDPC; i.e., both of them are extraordinary. Unlike this, if $\mu(\mathbf{r}) \equiv 1$, Eq. (2) has higher symmetry. As a result, the $E$ mode becomes the ordinary mode with $n_{\text {eff }}$ independent on $\hat{\mathbf{k}}[8]$. 
To obtain a homogenized equation from Eq. (3), we apply the plane-wave method that was developed in Ref. [8] for 2D nonmagnetic photonic crystal. Using the Bloch theorem and the periodicity of the functions $\varepsilon(\mathbf{r})$ and $\mu(\mathbf{r})$, we get the Fourier expansions:

$$
\begin{gathered}
H(\mathbf{r})=\exp (i \mathbf{k} \cdot \mathbf{r}) \sum_{\mathbf{G}} h_{k}(\mathbf{G}) \exp (i \mathbf{G} \cdot \mathbf{r}), \\
\varepsilon^{-1}(\mathbf{r})=\eta(\mathbf{r})=\sum_{\mathbf{G}} \eta(\mathbf{G}) \exp (i \mathbf{G} \cdot \mathbf{r}), \\
\mu^{-1}(\mathbf{r})=\xi(\mathbf{r})=\sum_{\mathbf{G}} \xi(\mathbf{G}) \exp (i \mathbf{G} \cdot \mathbf{r}),
\end{gathered}
$$

where $\mathbf{G}$ are the reciprocal-lattice vectors. Substituting Eq. (4) into Eq. (3), we get a generalized eigenvalue problem in $\mathbf{G}$ space:

$$
\begin{aligned}
& \sum_{\mathbf{G}^{\prime}} \eta\left(\mathbf{G}-\mathbf{G}^{\prime}\right)(\mathbf{k}+\mathbf{G}) . \\
& \quad\left(\mathbf{k}+\mathbf{G}^{\prime}\right) h_{k}\left(\mathbf{G}^{\prime}\right)=(\omega / c)^{2} \sum_{\mathbf{G}^{\prime}} \mu\left(\mathbf{G}-\mathbf{G}^{\prime}\right) h_{k}\left(\mathbf{G}^{\prime}\right) .
\end{aligned}
$$

The dispersion relation $\omega=\omega_{n}(\mathbf{k})(n=1,2, \ldots)$ is obtained from the condition that the set of Eq. (5) has a nontrivial solution.

It is easy to get from Eq. (5) that in the static limit, $\omega=k=0$, the Fourier coefficients $h_{k}(\mathbf{G} \neq 0)$ vanish. The only nonvanishing component is $h_{k}(\mathbf{G}=0)=h_{0}$. In the long-wavelength limit, $k a \ll 1$, this component determines the amplitude of the plane-wave solution, which is obtained from the first Eq. (4) by separating the term with $\mathbf{G}=0$ :

$$
H(\mathbf{r}) \approx h_{0} \exp (i \mathbf{k} \cdot \mathbf{r})+\sum_{\mathbf{G} \neq 0} h_{k}(\mathbf{G}) \exp (i \mathbf{G} \cdot \mathbf{r}) .
$$

Here we neglected $\mathbf{k}$ in the exponent in the correction term. Since the sun over $\mathbf{G}$ vanishes when $k \rightarrow 0$, Eq. (6) means that the medium homogenizes; i.e., the solution of the wave Eq. (3) approaches the plane wave.

Now the eigenvalue problem Eq. (5) can be solved using a perturbation theory with respect to small parameter $k a$. In the lowest approximation, we obtain

$$
\mathbf{k} \cdot \mathbf{G} \eta(\mathbf{G}) h_{0}+\sum_{\mathbf{G}^{\prime} \neq 0} \mathbf{G} \cdot \mathbf{G}^{\prime} \eta\left(\mathbf{G}-\mathbf{G}^{\prime}\right) h_{k}\left(\mathbf{G}^{\prime}\right)=0 .
$$

It follows from Eq. (7) that $h_{k}(\mathbf{G}) \propto k$. To obtain the second independent equation for the unknown $h_{0}$ and $h_{k}(\mathbf{G})$, we put $\mathbf{G}=0$ in Eq. (5) and keep the $k^{2}$ terms:

$$
\left(\bar{\eta} k^{2}-\bar{\mu} \omega^{2} / c^{2}\right) h_{0}+\sum_{\mathbf{G}^{\prime} \neq 0} \mathbf{k} \cdot \mathbf{G}^{\prime} \eta\left(-\mathbf{G}^{\prime}\right) h_{k}\left(\mathbf{G}^{\prime}\right)=0 .
$$

Here $\bar{\eta} \equiv \eta(\mathbf{G}=0)$ and $\bar{\mu} \equiv \mu(\mathbf{G}=0)$ are the bulk average of $1 / \varepsilon(\mathbf{r})$ and $\mu(\mathbf{r})$, respectively. For a binary composite, $\quad \bar{\eta}=f / \varepsilon_{a}+(1-f) / \varepsilon_{b}$ and $\bar{\mu}=f \mu_{a}+$ $(1-f) \mu_{b}$, where $f$ is the filling fraction of the inclusions 023904-2 (material $a$ ). Eliminating $h_{0}$ from Eqs. (7) and (8) and using the definition (1), we get a set of equations valid in the limit $k, \omega \rightarrow 0$ :

$$
\begin{aligned}
& \left(\bar{\eta}-n_{\mathrm{eff}}^{-2} \bar{\mu}\right) \sum_{\mathbf{G}^{\prime} \neq 0} \mathbf{G} \cdot \mathbf{G}^{\prime} \eta\left(\mathbf{G}-\mathbf{G}^{\prime}\right) h_{k}\left(\mathbf{G}^{\prime}\right)- \\
& \quad \sum_{\mathbf{G}^{\prime} \neq 0}(\hat{\mathbf{k}} \cdot \mathbf{G})\left(\hat{\mathbf{k}} \cdot \mathbf{G}^{\prime}\right) \eta(\mathbf{G}) \eta\left(-\mathbf{G}^{\prime}\right) h_{k}\left(\mathbf{G}^{\prime}\right)=0 .
\end{aligned}
$$

Equation (9) has nonzero solution if

$$
\begin{aligned}
\underset{\mathbf{G}, \mathbf{G}^{\prime} \neq 0}{\operatorname{det}}\left[\left(\bar{\eta}-n_{\mathrm{eff}}^{-2} \bar{\mu}\right) \mathbf{G} \cdot \mathbf{G}^{\prime} \eta\left(\mathbf{G}-\mathbf{G}^{\prime}\right)-\right. \\
\left.\left(\hat{\mathbf{k}} \cdot \mathbf{G}^{\prime}\right)\left(\hat{\mathbf{k}} \cdot \mathbf{G}^{\prime}\right) \eta(\mathbf{G}) \eta\left(-\mathbf{G}^{\prime}\right)\right]=0 .
\end{aligned}
$$

Although Eq. (10) is an infinite-order polynomial equation with respect to $\Lambda=\left(\bar{\eta}-n_{\text {eff }}^{-2} \bar{\mu}\right)$, it turns out that it has only a unique nonzero solution. To obtain this solution, we reduce Eq. (10) to a standard eigenvalue problem by multiplying it by the determinant of the inverse matrix, $\operatorname{det}\left\{\left[\mathbf{G} \cdot \mathbf{G}^{\prime} \eta\left(\mathbf{G}-\mathbf{G}^{\prime}\right)\right]^{-1}\right\}$ :

$$
\underset{\mathbf{G}, \mathbf{G}^{\prime} \neq 0}{\operatorname{det}}\left[B\left(\mathbf{G}, \mathbf{G}^{\prime}\right)-\Lambda \delta_{\mathbf{G G}^{\prime}}\right]=0,
$$

$$
\begin{aligned}
& B\left(\mathbf{G}, \mathbf{G}^{\prime}\right)=\hat{\mathbf{k}} \cdot \mathbf{G} \eta(\mathbf{G}) \sum_{\mathbf{G}^{\prime \prime} \neq 0} \hat{\mathbf{k}} \cdot \mathbf{G}^{\prime \prime} \\
& \times \eta\left(-\mathbf{G}^{\prime \prime}\right)\left[\mathbf{G}^{\prime \prime} \cdot \mathbf{G}^{\prime} \eta\left(\mathbf{G}^{\prime \prime}-\mathbf{G}^{\prime}\right)\right]^{-1} .
\end{aligned}
$$

The matrix $B\left(\mathbf{G}, \mathbf{G}^{\prime}\right)$ is a product of two factors, one of which depends only on $\mathbf{G}$ and the other only on $\mathbf{G}^{\prime}$. Then this matrix corresponds to a projection operator that has an eigenvalue $\Lambda=\operatorname{Tr} B\left(\mathbf{G}, \mathbf{G}^{\prime}\right)$. This results in the final formula for the index of refraction for the $H$ mode:

$$
\begin{aligned}
{\left[n_{\mathrm{eff}}^{(H)}(\hat{\mathbf{k}})\right]^{2}=\bar{\mu}\{} & \bar{\eta}-\sum_{\mathbf{G}, \mathbf{G}^{\prime} \neq \mathbf{0}}(\hat{\mathbf{k}} \cdot \mathbf{G})\left(\hat{\mathbf{k}} \cdot \mathbf{G}^{\prime}\right) \eta(\mathbf{G}) \\
& \left.\times \eta\left(-\mathbf{G}^{\prime}\right)\left[\mathbf{G} \cdot \mathbf{G}^{\prime} \eta\left(\mathbf{G}-\mathbf{G}^{\prime}\right)\right]^{-1}\right\}^{-1} .
\end{aligned}
$$

Equation (13) is valid for the arbitrary form of the unit cell, geometry of the inclusions, material composition of the photonic crystal, and direction of propagation in the plane of periodicity. The second factor $\{\cdots\}^{-1}$ coincides with the effective dielectric constant for a nonmagnetic photonic crystal. Therefore, in the case of $\mu=\bar{\mu}=1$, Eq. (13) is reduced to the result of Ref. [8].

The formula for $n_{\text {eff }}^{(E)}$ is obtained from Eq. (13) by the above mentioned interchange $\varepsilon$ by $\mu$ and vice versa.

$$
\begin{aligned}
{\left[n_{\mathrm{eff}}^{(E)}(\hat{\mathbf{k}})\right]^{2}=\bar{\varepsilon} } & \left\{\bar{\xi}-\sum_{\mathbf{G}, \mathbf{G}^{\prime} \neq 0}(\hat{\mathbf{k}} \cdot \mathbf{G})\left(\hat{\mathbf{k}} \cdot \mathbf{G}^{\prime}\right) \xi(\mathbf{G})\right. \\
& \left.\times \xi\left(-\mathbf{G}^{\prime}\right)\left[\mathbf{G} \cdot \mathbf{G}^{\prime} \xi\left(\mathbf{G}-\mathbf{G}^{\prime}\right)\right]^{-1}\right\}^{-1} .
\end{aligned}
$$

Equation (14) is reduced to $\left[n_{\text {eff }}^{(E)}\right]^{2}=\bar{\varepsilon}$ for nonmagnetic photonic crystal [8] when $\bar{\xi}=1$ and $\xi(\mathbf{G})=0$.

Transformation that occurs with the effective index of refraction for the $H$ mode in a MDPC is not very drastic.

023904-2 
It acquires an additional factor $\bar{\mu}$, which is obviously the effective permeability for the case when magnetic field is parallel to the cylinders. The directional dependence remains the same as it is for nonmagnetic photonic crystal. Unlike this, the $E$ mode undergoes qualitative transformation since the magnetic component of this mode lies in the plane of periodicity, and thus it is strongly affected by inhomogeneous magnetic medium. The effective index of refraction becomes $\hat{\mathbf{k}}$ dependent; i.e., this mode becomes extraordinary. The angular dependence of $n_{\text {eff }}$ for both modes can be easily obtained from Eqs. (13) and (14):

$$
n_{\mathrm{eff}}^{-2}(\hat{\mathbf{k}})=A_{i j} \hat{k}_{i} \hat{k}_{j}, \quad i, j=x, y
$$

For the $H$ mode, tensor $A_{i j}$ is given by

$$
\begin{aligned}
A_{i j}^{(H)}= & (\bar{\eta} / \bar{\mu}) \delta_{i j}-(1 / 2 \bar{\mu}) \sum_{\mathbf{G}, \mathbf{G}^{\prime} \neq 0}\left(G_{i} G_{j}^{\prime}+G_{j} G_{i}^{\prime}\right) \\
& \times \eta(\mathbf{G}) \eta\left(-\mathbf{G}^{\prime}\right)\left[\mathbf{G} \cdot \mathbf{G}^{\prime} \eta\left(\mathbf{G}-\mathbf{G}^{\prime}\right)\right]^{-1} .
\end{aligned}
$$

For the $E$ mode one needs to interchange dielectric and magnetic constants in Eq. (16). Equation (15) describes an ellipse in the $x-y$ plane, which means that both modes are extraordinary waves. This situation does not exist in natural crystals, where one of the polarizations always exhibits isotropy when the wave vector sweeps a plane. The reason for this universal symmetry is due to the fact that the fundamental equation of crystal optics - the Fresnel equation-is derived assuming that atoms of a transparent medium are nonmagnetic [3]. This assumption is not applicable for MDPCs. In magnetically anisotropic medium, Fresnel's equation is replaced by the following one:

$$
\begin{gathered}
\left(\frac{\hat{n}_{x}^{2}}{\mu_{y} \mu_{z}}+\frac{\hat{n}_{y}^{2}}{\mu_{x} \mu_{z}}+\frac{\hat{n}_{z}^{2}}{\mu_{x} \mu_{y}}\right)\left(\varepsilon_{x} \hat{n}_{x}^{2}+\varepsilon_{y} \hat{n}_{y}^{2}+\varepsilon_{z} \hat{n}_{z}^{2}\right)- \\
\varepsilon_{x}\left(\frac{\varepsilon_{y}}{\mu_{y}}+\frac{\varepsilon_{z}}{\mu_{z}}\right) \hat{n}_{x}^{2}-\varepsilon_{y}\left(\frac{\varepsilon_{x}}{\mu_{x}}+\frac{\varepsilon_{z}}{\mu_{z}}\right) \hat{n}_{y}^{2}-\varepsilon_{z}\left(\frac{\varepsilon_{x}}{\mu_{x}}+\frac{\varepsilon_{y}}{\mu_{y}}\right) \hat{n}_{z}^{2}+\varepsilon_{x} \varepsilon_{y} \varepsilon_{z}=0 .
\end{gathered}
$$

Here $\mathbf{n}=(k c / \omega) \hat{\mathbf{k}}$, and we assumed that the tensors $\varepsilon_{i k}$ and $\mu_{i k}$ have common principal axes.

This fourth-order equation defines the wave-vector surface. The surface has four points of self-intersection as well as in the case of nonmagnetic atoms. One octant of this surface is shown in Fig. 1. Intersection of the surface with the $x-y$ plane $\left(n_{z}=0\right)$ occurs along two ellipses:

$$
n_{x}^{2} /\left(\varepsilon_{z} \mu_{y}\right)+n_{y}^{2} /\left(\varepsilon_{z} \mu_{x}\right)=1,
$$

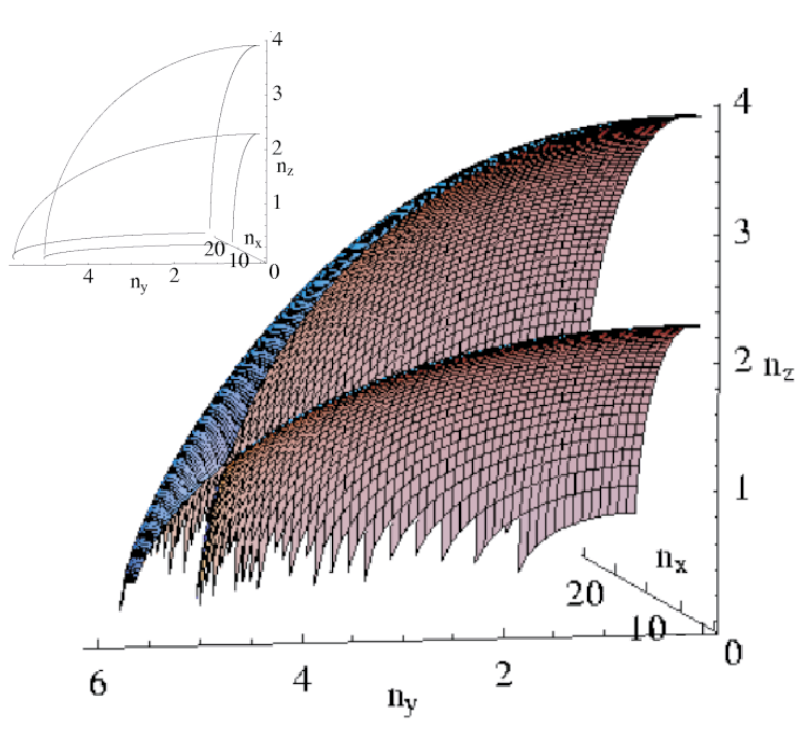

FIG. 1 (color online). One octant of the wave-vector surface Eq. (17) drawn for $\varepsilon_{x}=1, \varepsilon_{y}=4, \varepsilon_{z}=25, \mu_{x}=1.3, \mu_{y}=$ 15.3 , and $\mu_{z}=25$. The point of self-intersection lies in the $x-y$ plane. The inset shows the projections on the coordinate planes.

$$
n_{x}^{2} /\left(\varepsilon_{y} \mu_{z}\right)+n_{y}^{2} /\left(\varepsilon_{x} \mu_{z}\right)=1
$$

To establish a correspondence between these two ellipses and the $E$ and $H$ modes of a 2D MDPC, we take into account that in a nonmagnetic medium the ellipse (18) becomes a circle; i.e., the corresponding mode becomes ordinary wave. It is clear that this ellipse corresponds to the $E$ mode which is known to be an ordinary wave in a nonmagnetic photonic crystal [8]. Then the ellipse (19) corresponds to the $H$ mode. The principal components of the tensors $\varepsilon_{i k}$ and $\mu_{i k}$ can be obtained from Eqs. (15) and (16). The products $1 /\left(\varepsilon_{z} \mu_{y}\right)$ and $1 /\left(\varepsilon_{z} \mu_{x}\right)$ are the principal values of the tensor $A_{i k}^{(E)}$. Since for the $E$ mode $\varepsilon_{z}=\bar{\varepsilon}$, the inverse permeabilities in the plane of periodicity, $\mu_{y}$ and $\mu_{x}$, are the eigenvalues of the tensor $\bar{\varepsilon} A_{i k}^{(E)}$. Similarly, considering Eq. (19), we obtain that $1 / \varepsilon_{x}$ and $1 / \varepsilon_{y}$ are the eigenvalues of the tensor $\bar{\mu} A_{i k}^{(H)}$ and $\mu_{z}=\bar{\mu}$. Thus all the components of the tensors $\varepsilon_{i k}$ and $\mu_{i k}$ can be obtained from Eqs. (15) and (16). These equations together with the modified Fresnel equation (17) are the fundamental equations of the optics of MDPCs.

Using tensors $\varepsilon_{i k}$ and $\mu_{i k}$, the tensor of surface impedance $Z_{i k}$ can be introduced. Its principal values are $Z_{i}=$ $\sqrt{\mu_{i} / \varepsilon_{i}}$. This tensor determines the reflection coefficient from homogeneous anisotropic medium. Recently, the effective surface impedance was calculated for 2D MDPCs at frequencies close to the Mie resonance [9].

Since for the MDPCs both polarizations turn out to be extraordinary waves, the index (or Fresnel) ellipsoid cannot be introduced. In nonmagnetic crystals the index ellipsoid determines (for each direction of $\hat{\mathbf{k}}$ ) two values 
of the index of refraction $n$ for two independent polarizations and also two orthogonal directions of the displacement vector $\mathbf{D}$. If the vector $\hat{\mathbf{k}}$ sweeps a plane, one of the values of $n$ remains unchanged. Equations (18) and (19) show that this is not the case for the MDPCs. Leaving the details for a complete publication, we mention here that for arbitrary direction $\hat{\mathbf{k}}$ the values of $n$ are given by the principal values of a two-dimensional tensor

$$
n_{\alpha \beta}=\frac{\left(\hat{\mu} \hat{\varepsilon}^{-1}\right)_{\alpha \beta}}{\operatorname{det} \hat{\mu}} .
$$

Here $\alpha, \beta=1,2$ label two orthogonal directions $\mathbf{u}_{1}, \mathbf{u}_{2}$ in the plane perpendicular to $\hat{\mathbf{k}}$. Tensors $\hat{\varepsilon}$ and $\hat{\mu}$ are projections of the corresponding three-dimensional tensors on this plane, i.e., $\varepsilon_{\alpha \beta}=\varepsilon_{i k} u_{i}^{(\alpha)} u_{k}^{(\beta)}$ and $\mu_{\alpha \beta}=$ $\mu_{i k} u_{i}^{(\alpha)} u_{k}^{(\beta)}$. The directions of the displacement vector $\mathbf{D}$ for two independent polarizations coincide with principal axes of the tensor $n_{\alpha \beta}$.

Having calculated all the homogenized parameters of a MDPC, let us compare the results for $n_{\text {eff }}$ obtained from static and dynamic approaches. In this Letter we are using dynamic approach since we calculate the limit (1) from the Maxwell equations. According to Eqs. (13) and (14), the index of refraction for the two polarizations is written as follows:

$$
n_{\mathrm{eff}}^{(E)}=\sqrt{\bar{\varepsilon} \mu_{\mathrm{st}}}, \quad \text { and } \quad n_{\mathrm{eff}}^{(H)}=\sqrt{\varepsilon_{\mathrm{st}} \bar{\mu}} .
$$

Here $\varepsilon_{\mathrm{st}}$ and $\mu_{\mathrm{st}}$ are the statics values of the effective permeability and permittivity. They are given by the expressions in curly bracket in Eqs. (13) and (14), respectively. These quantities can be calculated using either electrostatic or magnetostatic approach. However, $n_{\text {eff }}$ cannot be calculated using only one of these approaches. This is because in the electrostatic (magnetostatic) approach magnetic (dielectric) properties of matter are completely ignored. Static and dynamic approaches coincide only for structures with $\bar{\varepsilon}=1(\bar{\mu}=1)$.

A particular case of perfectly conducting cylinders, $\epsilon_{a} \rightarrow \infty$, requires special attention. In this case, $n_{\mathrm{eff}}^{(E)}=$ $\infty$; therefore the $E$ mode does not exist. At finite frequencies the magnetic field does not penetrate into a perfect conductor, and it behaves like a superconductor, i.e., ideal diamagnetic, with $\mu_{a}=0$. Then $\bar{\mu}=1-f$ and it follows from Eq. (21) that

$$
n_{\mathrm{eff}}^{(H)}=\sqrt{(1-f) \varepsilon_{\mathrm{st}}} .
$$

Here $\varepsilon_{\text {st }}$ depends only on the filling fraction $f$. An explicit formula for $\varepsilon_{\text {st }}$ for square lattice and circular cylinders is given in Ref. [10]. It was shown there that this formula is in complete agreement with numerical results obtained much earlier [11] using static approach. A photonic band structure for a PC of perfectly conducting cylinders has been calculated numerically in Ref. [12]. The effective dielectric constant was evaluated from the slope of the acoustic band at $k \rightarrow 0$. This dynamic dielectric constant $\varepsilon_{\mathrm{dyn}}$ turned out to be different from static value $\varepsilon_{\mathrm{st}}$. It was claimed in Ref. [12] that this difference is due to the noncommuting limits $\epsilon_{a} \rightarrow \infty$ and $k \rightarrow 0$. In a recent publication, Felbacq [13] gave a rigorous proof that $\varepsilon_{\mathrm{dyn}}=(1-f) \varepsilon_{\mathrm{st}}$ for an array of circular cylinders arranged in a square lattice and also attributed this to the noncommuting limits. Now it is clear from Eq. (22) that the case of perfectly conducting cylinders is not anomalous and the limits do commute. The factor $1-f$ in Eq. (22), which causes the discrepancy, is the average magnetic constant of the structure. It is worthwhile to mention that the dynamic dielectric constant $\varepsilon_{\mathrm{dyn}}$, being a product of magnetic and dielectric constants, does not have physical meaning. The effective index of refraction (22) should be considered instead. Equation (22) in not valid for metallic cylinders where $\left|\varepsilon_{a}\right| \propto \omega^{-1}$. Results for this special case will be published elsewhere.

In conclusion, in this Letter we demonstrated that 2D periodic magnetodielectric structure homogenizes at low frequencies and calculated the low-frequency index of refraction. Unlike pure dielectric structures, MDPC exhibits unusual optical anisotropy. Both polarizations, $E$ and $H$ modes, are extraordinary waves with unusual angular dependence of the speed of light. The effects of birefringence and conical refraction require special study since they apparently manifest themselves in a way different from that in natural crystals.

This work is supported by CONACyT Grant No. 42136F. The work has been completed in ICTP (Italy). A. A. K. is thankful to the ICTP for hospitality and to the members of the Condensed Matter Group for useful discussions.

[1] M. M. Sigalas et al., Phys. Rev. B 56, 959 (1997).

[2] C.-S. Kee et al., Phys. Rev. B 61, 15523 (2000).

[3] L. D. Landau, E. M. Lifshitz, and L. P. Pitaevskii, Electrodynamics of Continuous Media (Pergamon, Oxford, 1984), 2nd ed..

[4] D. R. Smith and D. Schurig, Phys. Rev. Lett. 90, 077405 (2003).

[5] L. V. Panina et al., Phys. Rev. B 66, 155411 (2002).

[6] F. X. Redl et al., Nature (London) 423, 968 (2003); Y. Saado et al., Phys. Rev. B 66, 195108 (2003).

[7] I. L. Lyubchanskii et al., J. Phys. D 36, R277 (2003).

[8] P. Halevi et al., Phys. Rev. Lett. 82, 719 (1999); A. A. Krokhin et al., Phys. Rev. B 65, 115208 (2002).

[9] S. O’Brien and J. B. Pendry, J. Phys. Condens. Matter 14, 4035 (2002).

[10] P. Halevi, A. A. Krokhin, and J. Arriaga, Phys. Rev. Lett. 86, 3211 (2001).

[11] W.T. Perrins et al., Proc. R. Soc. London A 369, 207 (1979).

[12] N. A. Nicorovici et al., Phys. Rev. Lett. 75, 1507 (1995); Phys. Rev. E 52, 1135 (1995); R. C. McPhedran et al., J. Electromagn. Waves. Appl. 11, 981 (1997).

[13] D. Felbacq, J. Math. Phys. 43, 52 (2002); J. Phys. A 33, 815 (2000). 